\title{
No Association Between Intrauterine Contraceptive Devices and Musculoskeletal Hip Joint Pain
}

\author{
Nathan H. Varady, M.D., M.B.A., Paul Abraham, M.D., Michael P. Kucharik, B.S., \\ Christopher T. Eberlin, B.S., David Freccero, M.D., Eric L. Smith, M.D., and \\ Scott D. Martin, M.D.
}

\begin{abstract}
Purpose: To investigate the association between intrauterine device (IUD) use and hip pain, orthopaedic visits for hip pain, and arthroscopic hip surgery. Methods: This was a retrospective cohort study of patients aged $18-44$ years old using either IUDs or subdermal implants for contraception in a large commercial claims database (MarketScan) from 2012 to 2015. All patients had at least 12 months of continuous enrollment both before and after contraceptive placement. Patients with a history of hip pain or surgery were excluded. The primary outcome was new hip pain. Secondary outcomes included visiting an orthopaedic or sports medicine provider for a hip complaint, intra-articular hip injection, and arthroscopic hip surgery. Outcomes were analyzed with Cox proportional-hazard models. Results: We identified a total of 242,383 patients, including $216,541(89.3 \%)$ with IUDs and 25,842 (10.7\%) with subdermal contraceptive implants. In time-to-event analysis, IUDs (vs implants) were not associated with increased risk of new hip pain diagnoses (hazard ratio [HR] 0.95, 95\% confidence interval [CI] 0.87-1.03, $P=.21)$. In contrast, both age $(P<.001)$ and region $(P<.001)$ were associated with increased risk of new hip pain. Similar results were seen for the secondary outcomes, including risk of orthopaedic visits for hip complaints (HR 1.06, 95\% CI 0.83-1.35, $P=.63$ ), intra-articular injections of the hip (HR 0.94, 95\% CI 0.63-1.41, $P=.77$ ), and hip arthroscopy procedures (HR 1.13, 95\% CI 0.53-2.40, $P=.75$ ). Conclusions: In this study, we found no evidence that IUDs were associated with hip pain or surgery. Level of Evidence: Level III, retrospective cohort.
\end{abstract}

$\mathbf{T}$ he number of patients presenting with hip pain concerning for acetabular labral pathology and/or femoroacetabular impingement is on the rise. ${ }^{1-6}$ While the etiology of these patients' pain often can be ascertained with physical examination and diagnostic imaging, there remains a considerable number of patients for whom the source of symptoms remains unclear. ${ }^{7-13}$

From the Department of Orthopaedic Surgery, Massachusetts General Hospital/Harvard Medical School (N.H.V., P.A., M.P.K., C.T.E., S.D.M.); Department of Orthopaedic Surgery, Boston Medical Center (D.F.); and Department of Orthopaedic Surgery, New England Baptist Hospital (E.L.S.), Boston, Massachusetts, U.S.A.

The authors report that they have no conflicts of interest in the authorship and publication of this article. Full ICMJE author disclosure forms are available for this article online, as supplementary material.

Received February 26, 2021; accepted July 13, 2021.

Address correspondence to Michael P. Kucharik, B.S., Department of Orthopaedic Surgery, Massachusetts General Hospital/Harvard Medical School, Boston, MA 02114.E-mail:mikekucharik@gmail.com

(C) 2021 THE AUTHORS. Published by Elsevier Inc. on behalf of the Arthroscopy Association of North America. This is an open access article under the CC BY-NC-ND license (http://creativecommons.org/licenses/by-nc-nd/4.0/).

2666-061X/21134

https://doi.org/10.1016/j.asmr.2021.07.003
Moreover, radiologic musculoskeletal abnormalities are present in as many as $50 \%$ of asymptomatic, prearthritic individuals. ${ }^{10,14-18}$ Thus, the appropriate correlation of imaging abnormalities and patient symptomatology is a challenge presented to physicians and speaks to the art of clinical medicine.

These challenges can be particularly pronounced among premenopausal women due to the number of gynecologic etiologies that can mimic musculoskeletal disease. For instance, ovarian cysts, pelvic inflammatory disease, pelvic adhesions, leiomyomata, adenomyosis, and endometriosis, among others, can all present similarly to musculoskeletal hip pathology in some patients. ${ }^{8,9,19-21}$ In most cases, these symptoms arise secondary to the pelvic inflammation associated with these conditions. ${ }^{12,19,22-24}$ To avoid unnecessary arthroscopic hip surgery, it is thus critical to identify and/or rule out all possible gynecologic causes of hip pain.

Interestingly, although local pelvic inflammation states are a well-known source of potential hip pain, ${ }^{12,19,22-24}$ intrauterine devices (IUDs), which predominantly feature an inflammatory mechanism of 
Table 1. Baseline demographic factors overall and between patients with intrauterine devices (IUDs) and subdermal implant contraceptives.

\begin{tabular}{|c|c|c|c|c|}
\hline & \multicolumn{2}{|c|}{ Contraceptive Type } & \multirow{3}{*}{$\begin{array}{c}\text { Total } \\
(\mathrm{N}=242,383)\end{array}$} & \multirow[b]{3}{*}{ P Value } \\
\hline & IUD & Implant & & \\
\hline & $(\mathrm{N}=216,541)$ & $(\mathrm{N}=25,842)$ & & \\
\hline \multicolumn{5}{|l|}{ Age } \\
\hline Mean (SD) & $32.1(6.9)$ & $24.6(5.8)$ & $31.3(7.2)$ & $<0.001$ \\
\hline $18-24$ & $37444(17.3 \%)$ & $16298(63.1 \%)$ & $53742(22.2 \%)$ & $<0.001$ \\
\hline $25-34$ & $94758(43.8 \%)$ & $7523(29.1 \%)$ & $102281(42.2 \%)$ & \\
\hline $35-44$ & $84339(38.9 \%)$ & $2021(7.8 \%)$ & $86360(35.6 \%)$ & \\
\hline \multicolumn{5}{|l|}{ Region } \\
\hline North Central & $51242(23.7 \%)$ & $6902(26.7 \%)$ & $58144(24.0 \%)$ & $<0.001$ \\
\hline Northeast & $33012(15.2 \%)$ & $2359(9.1 \%)$ & $35371(14.6 \%)$ & \\
\hline South & $72271(33.4 \%)$ & $11148(43.1 \%)$ & $83419(34.4 \%)$ & \\
\hline West & $54286(25.1 \%)$ & $4643(18.0 \%)$ & $58929(24.3 \%)$ & \\
\hline Unknown & $5730(2.6 \%)$ & $790(3.1 \%)$ & $6520(2.7 \%)$ & \\
\hline \multicolumn{5}{|l|}{ year } \\
\hline 2011 & $47868(22.1 \%)$ & $1661(6.4 \%)$ & $49529(20.4 \%)$ & $<0.001$ \\
\hline 2012 & $55864(25.8 \%)$ & $6016(23.3 \%)$ & $61880(25.5 \%)$ & \\
\hline 2013 & $63145(29.2 \%)$ & $9025(34.9 \%)$ & $72170(29.8 \%)$ & \\
\hline 2014 & $49664(22.9 \%)$ & $9140(35.4 \%)$ & $58804(24.3 \%)$ & \\
\hline
\end{tabular}

$\mathrm{SD}=$ standard deviation; $\mathrm{y}=$ years

action, are unknown to be a cause for hip pain in some prearthritic women. ${ }^{25-27}$ This question is of particular importance, due to the growing number of women using IUDs as their method of contraception, now the most common form of contraception in the world. ${ }^{28-30}$ In fact, it is possible that some of the increasing burden of hip pain and resultant arthroscopic hip surgery in women could be explained by the rising rates of IUD use. The purpose of this study was to investigate the association between IUD use and hip pain, orthopaedic visits for hip pain, and arthroscopic hip surgery. We hypothesized that patients with IUDs would report greater rates of hip pain and surgery compared with patients with other forms of contraception. Second, we hypothesized that any direct hip or pelvic pain causing effect, if present, may be more pronounced for those with copper IUDs due to their direct inflammatory mechanism of action..$^{25,26}$

\section{Methods}

We used the Truven MarketScan Commercial Claims and Encounters Database from January 1, 2011, to September 30, 2015, for this study. The MarketScan database is a premier commercial-claims database with data from more than 350 unique carriers. The database has several key strengths, including the availability of granular data for all inpatient and outpatient services, longitudinal patient tracking, detailed enrollment files to exclude patients who may have lost/switched insurance coverage and fallen out of the database, and comprehensive outpatient drug information. The database contains more than 250 million unique patients and had at least 40 million covered individuals each year of the study period.

This was a retrospective cohort study of patients 18-44 years old using either IUDs or subdermal implants for contraception. Subdermal contraceptive implants were chosen as a control group to serve as an active comparator to IUDs, given that they are another highly effective form of long-acting contraception that require a procedure to initiate. However, they are not local to the pelvic region (typically implanted in the arm), and therefore have no direct mechanism of causing hip pain based on location alone. By using a control group with similar indication and patient predilection, we help to minimize unmeasured confounders. For all patients, their most recent contraceptive method was examined, and the date of insertion was considered the index date. All patients had to have at least 12 months of continuous enrollment both before and after contraceptive placement. This was required so that baseline patient factors could be collected over the year before contraception placement, as well as to require at least some amount of time for hip-related outcomes to develop. Critically, any patients with any evidence of hip pain or surgery in the year before contraceptive placement were excluded, as were patients who used both forms of contraception within a year of the index initiation.

Additional demographic factors collected included age, geographic region, contraceptive use history (including previous use of oral contraceptives, implants, and IUDs), year of insertion, and medical comorbidities. To assess the association between IUD placement and 


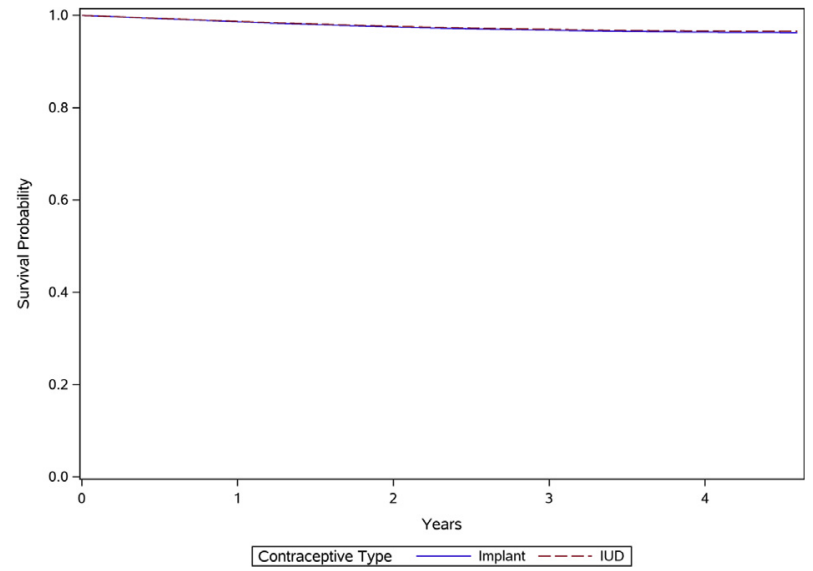

Fig 1. Survival curves for time remaining free of hip pain after insertion of either an intrauterine device (IUD) or subdermal implant for contraception. The appearance of only one curve is due to the substantial overlap in hip pain-free survival between contraceptive methods.

potential spurious musculoskeletal hip issues, we examined 4 outcomes. The primary outcome was the development of hip joint pain. Secondary outcomes included visiting an orthopaedic or sports medicine provider for hip pain, undergoing intra-articular hip injection, and undergoing arthroscopic hip surgery. Patients were followed until the occurrence of each respective outcome or database drop out. In addition, we performed a sensitivity analysis comparing copper versus hormonal IUDs and implants. Although both categories of IUDs (copper and hormonal) primarily work through a foreign body effect, causing a local inflammatory reaction that is toxic to sperm and ova and impairing of implantation, ${ }^{25-27}$ the copper IUD is believed to provide additional contraceptive benefit by increasing the inflammatory response, ${ }^{25,26}$ whereas hormonal IUDs may help thicken cervical mucus resulting in blockage of the upper genital tract. ${ }^{25,31}$ As such, we hypothesized that any direct hip or pelvic pain causing effect, if present, may be more pronounced for those with copper IUDs.

\section{Statistical Analysis}

Baseline patient demographic factors were presented as $\mathrm{n}(\%)$ and mean (standard deviation) and compared with $\chi^{2}$ or $t$ tests, as appropriate. Outcomes were analyzed using a time-to-event approach with Cox-proportional hazard models. In addition to the exposure of interest (contraceptive method), Cox models were adjusted for age, geographic region, and year of insertion. Censoring events are described as mentioned previously. Adjusted survival curves are plotted at the reference levels observed in the data. All statistical analyses were performed in SAS, version 9.4 (SAS Institute, Cary, NC), $P$ values were 2 -sided, and $P<.05$ was considered significant.
Given this database contains only deidentified, Health Insurance Portability and Accountability Act-compliant information, this study was exempt from institutional review board approval (Partners Protocol \#2019P001872).

\section{Results}

We identified a total of 242,383 patients, including $216,541(89.3 \%)$ with IUDs and 25,842 (10.7\%) with subdermal contraceptive implants. Mean (standard deviation) age across all patients was 31.3 (7.2) years. In general, subdermal implant patients were younger and more likely to live in the South and North Central United States, whereas IUDs were common in the West (Table 1). In addition, the relative use of implants was more common as time progressed. Overall, 7,688 $(3.2 \%)$ patients experienced hip pain in the follow-up period, $1,175(0.5 \%)$ saw an orthopaedic surgeon or sports medicine specialist for that pain, $427(0.2 \%)$ underwent intra-articular hip injection, and $92(0.0 \%)$ underwent hip arthroscopy.

In time-to-event analysis adjusting for age, geographic region, and calendar year, IUDs (vs implants) were not associated with increased risk of hip pain diagnoses (hazard ratio [HR] $0.95,95 \%$ confidence interval [CI] 0.87-1.03, $P=.21$ ) (Fig 1). In contrast, increasing age (HR 1.03 per additional year, 95\% CI 1.03-1.04, $P<.001$ ) and region (Northeast vs South: HR 1.26, 95\% CI 1.17-1.34, $P<.001)$ were associated with risk of hip pain.

Similar results were seen for the secondary outcomes. Specifically, IUDs, relative to subdermal implants, were not associated with increased risk of orthopaedic visits for hip pain (HR 1.06, 95\% CI 0.83-1.35, $P=.63$ ), intra-articular injections of the hip (HR 0.94, 95\% CI 0.63-1.41, $P=.77$ ), or hip arthroscopy procedures (HR $1.13,95 \%$ CI $0.53-2.4, P=.75)$.

In sensitivity analyses comparing copper versus hormonal IUDs and subdermal implants, copper IUDs were associated with a slightly lower risk of hip pain. Specifically, there was lower risk of developing hip pain in patients with copper IUDs versus hormonal IUDs (HR $0.94,95 \%$ CI $0.88-0.999, P=.048$ ) and copper IUDs versus subdermal implants (HR 0.90, 95\% CI 0.81-0.995, $P=.04$ ), whereas there was no significant difference in risk of developing hip pain between hormonal IUDs and subdermal implants (HR 0.96, 95\% CI $0.88-1.05, P=.33$ ).

\section{Discussion}

The most important finding of this study was the lack of association between IUDs and hip pain, which was in contrast to our hypothesis. Identifying and ruling out non-musculoskeletal causes of patients' symptoms is critical to avoiding unnecessary orthopaedic surgery. While there are many gynecologic pathologies that can 
occasionally present with symptoms consistent with musculoskeletal hip pain, whether contraceptive IUDs are associated with this clinical presentation was unknown. In this study, we found no evidence that IUDs are associated with hip pain or surgery compared with another common implantable contraceptive method.

Specifically, we found no difference in rates of hip arthroscopy, intra-articular hip injections, visits with orthopaedic or sports medicine providers for hip complaints, or any hip pain diagnoses, when compared with an implant control group. We chose patients with contraceptive implants as our control group because, like IUDs, contraceptive implants are implantable longacting contraceptive devices but not local to the pelvic region and would therefore have no direct mechanism of causing a local inflammatory reaction that could lead to hip pain. Despite disproving our initial hypothesis, the aforementioned findings are useful to both the orthopaedic surgeon and gynecologist in their evaluation of hip pain of unknown etiology in premenopausal women. Moreover, we found that increasing age and region were significantly associated with risk of hip pain. Increasing age and its association with hip pain is unsurprising, as increased age has been shown to be associated with an increased incidence of osteoarthritis. $^{32-34}$ As for regional bias, the Northeast region was associated with increased risk of hip pain, which is likely due to access of health care. ${ }^{35,36}$

To explore potential hormonal/systemic effects, we then compared copper IUDs with hormonal IUDs and hormonal subdermal implants. Interestingly, in this analysis, we found a very small reduction in risk of hip pain for copper IUDs compared with the hormonal contraceptive methods. Multiple possibilities could explain these results, including treatment decisions or the hormonal effects themselves. For instance, while all patients had no hip pain for at least a year before the initiation date, it is possible that a small fraction of patients received hormonal rather than copper IUDs specifically due to their ability to treat other conditions that may be associated with hip pain (e.g., endometriosis $^{19,22}$ ). Alternatively, previous work has shown that hormonal contraception can be associated with pelvic girdle pain, ${ }^{37,38}$ which could be presenting as hip pain for a small number of patients. Consistent with this possibility, we found no difference in risk of hip pain between subdermal implants and hormonal IUDs (i.e., hormonal vs hormonal), while copper IUDs had significantly lower risk of hip pain compared with each of the hormonal methods. While the effect sizes are small, and these findings appear to be unrelated to the IUDs themselves, future work should continue to investigate the role (if any) of hormonal influences in the development of musculoskeletal pain. ${ }^{39-41}$ While evidence directly linking hormonal contraceptives with musculoskeletal pain is limited, several studies have shown a possible correlation between hormonal fluctuations and development of pain. ${ }^{39-41}$

Anecdotally, we had seen many young women with IUDs and symptoms highly consistent with acetabular labral pathology in our high-volume hip arthroscopy practice who went on to have unimpressive magnetic resonance imaging and/or arthroscopic findings. Given the well-established mechanisms of all IUDs to work by causing a local pelvic inflammatory state, ${ }^{25-27}$ it was highly plausible that IUDs may have been playing a role in some of these symptoms. Nevertheless, the finding that there was no association between IUDs and hip pain in this large, national sample is encouraging because it suggests that many young women are not receiving unnecessary arthroscopic hip surgery related to their contraceptive device.

Although IUDs do not appear to be a major contributor to hip pain, improving our diagnostic techniques and identifying potentially new etiologies of disease remains a vitally important task, particularly in young, active women, who suffer from both chronic pelvic pain and chronic hip pain syndromes in high rates. ${ }^{9}$ Since both of these syndromes have highly variable clinical presentations, ${ }^{9}$ it is important for orthopaedic specialists to rule out gynecologic causes of pain and for gynecologic specialists to rule out musculoskeletal causes of pain in this patient population. Nevertheless, anecdotally, many patients without classic presentations for either condition and unimpressive orthopaedic and gynecologic imaging continue to report to orthopaedic specialists with hip pain. This highlights the need for more research on clinical mimickers of hip pain, which has been noted to be a challenging diagnosis for orthopaedic surgeons, primary care physicians, and gynecologists alike. ${ }^{42-44}$

Arthroscopic hip surgery and IUDs are both becoming increasingly common. To avoid surgery that may ultimately prove unbeneficial to the patient, ruling out alternative etiologies of hip pain is paramount. While many gynecologic pathologies have the potential to imitate musculoskeletal symptoms in select patients, IUDs do not appear to be a major risk of false-positive musculoskeletal hip pain. Moving forward, IUDrelated symptomology may not need to be a major consideration in the differential diagnosis of hip joint pain.

\section{Limitations}

There are many aspects of the MarketScan database that make it an excellent method for studying this question: a very large, national sample to study the relatively rare outcome of hip arthroscopy; longitudinal tracking of patients with detailed records of all contraceptive methods; and a sample of commercially insured individuals, under which most women of childbearing age fall. Still, this study is not without limitations. First, 
it is a retrospective, observational study and subject to all limitations therein, including the possibility of uncontrolled confounders. However, by using an active comparator of similar indication, adjusted analyses, and an array of sensitivity analyses, the likelihood of our results being due to confounding is lessened. To address potential bias, we attempted to minimize selection bias by implementing a control group with similar indications without a mechanism for direct hip pain. However, it is always possible that differences between patients in both groups may confound results with unforeseen selection bias. Moreover, as with almost all databases, we were unable to review any written history or physical exam information, which would have been another way to mitigate selection bias. Next, we were focused only on potentially idiopathic hip pain that providers may have suspected to be of musculoskeletal nature. This study did not assess the relative safety of IUDs versus implants across all outcomes, and it is possible there may be differences in infection rates or other complications between approaches. Nevertheless, any such complications do not appear to mimic musculoskeletal pathology, which was the focus of the current work. Finally, due to incomparability of initiation dates and the inability to determine pill consumption, this study could not analyze oral contraceptives, which may be able to be investigated in future institutional studies.

\section{Conclusions}

In this study, we found no evidence that IUDs were associated with hip pain or surgery.

\section{References}

1. Bozic KJ, Chan V, Valone FH 3rd, Feeley BT, Vail TP. Trends in hip arthroscopy utilization in the United States. J Arthroplasty 2013;28:140-143.

2. Colvin AC, Harrast J, Harner C. Trends in hip arthroscopy. J Bone Joint Surg Am 2012;94:e23.

3. Cvetanovich GL, Chalmers PN, Levy DM, et al. Hip arthroscopy surgical volume trends and 30-day postoperative complications. Arthroscopy 2016;32:1286-1292.

4. Kivlan BR, Nho SJ, Christoforetti JJ, et al. Multicenter outcomes after hip arthroscopy: Epidemiology (MASH Study Group). What are we seeing in the office, and who are we choosing to treat? Am J Orthop (Belle Mead NJ) 2017;46:35-41.

5. Montgomery SR, Ngo SS, Hobson T, et al. Trends and demographics in hip arthroscopy in the United States. Arthroscopy 2013;29:661-665.

6. Zhang AL, Feeley BT. Editorial Commentary: The rise of hip arthroscopy: Temporary trend or here to stay? Arthroscopy 2018;34:1831-1832.

7. Bhatia A, Hoydonckx Y, Peng P, Cohen SP. Radiofrequency procedures to relieve chronic hip pain: An evidence-based narrative review. Reg Anesth Pain Med 2018;43:72-83.
8. Gyang A, Hartman M, Lamvu G. Musculoskeletal causes of chronic pelvic pain: What a gynecologist should know. Obstet Gynecol 2013;121:645-650.

9. Lillemon JN, Nardos R, Kaul MP, Johnson AN, Choate A, Clark AL. Complex Female pelvic pain: A case series from a multidisciplinary clinic in urogynecology and physiatry. Female Pelvic Med Reconstr Surg 2019;25:e34-e39.

10. Prather H, Hunt D, Rho M, Yemm T, Fong K, Brophy RH. Abnormal hip physical examination findings in asymptomatic female soccer athletes. Knee Surg Sports Traumatol Arthrosc 2015;23:2106-2114.

11. Retchford TH, Tucker KJ, Weinrauch P, et al. Clinical features of people with hip-related pain, but no clinical signs of femoroacetabular impingement syndrome. Phys Ther Sport 2018;34:201-207.

12. Speer LM, Mushkbar S, Erbele T. Chronic pelvic pain in women. Am Fam Physician 2016;93:380-387.

13. Uding A, Bloom NJ, Commean PK, et al. Clinical tests to determine femoral version category in people with chronic hip joint pain and asymptomatic controls. Musculoskelet Sci Pract 2019;39:115-122.

14. Diesel CV, Ribeiro TA, Scheidt RB, Macedo CA, Galia CR. The prevalence of femoroacetabular impingement in radiographs of asymptomatic subjects: A cross-sectional study. Hip Int 2015:25:258-263.

15. Frank JM, Harris JD, Erickson BJ, et al. Prevalence of femoroacetabular impingement imaging findings in asymptomatic volunteers: A systematic review. Arthroscopy 2015;31:1199-1204.

16. Gerhardt MB, Romero AA, Silvers HJ, Harris DJ, Watanabe D, Mandelbaum BR. The prevalence of radiographic hip abnormalities in elite soccer players. Am J Sports Med 2012;40:584-588.

17. Kapron AL, Peters CL, Aoki SK, et al. The prevalence of radiographic findings of structural hip deformities in female collegiate athletes. Am J Sports Med 2015;43: 1324-1330

18. Vahedi H, Aalirezaie A, Azboy I, Daryoush T, Shahi A, Parvizi J. Acetabular labral tears are common in asymptomatic contralateral hips with femoroacetabular impingement. Clin Orthop Relat Res 2019;477:974-979.

19. Giudice LC. Clinical practice. Endometriosis. N Engl J Med 2010;362:2389-2398.

20. Lamvu G, Carrillo J, Witzeman K, Alappattu M. Musculoskeletal considerations in female patients with chronic pelvic pain. Semin Reprod Med 2018;36:107-115.

21. Martin HD, Shears SA, Palmer IJ. Evaluation of the hip. Sports Med Arthrosc Rev 2010;18:63-75.

22. Lebovic DI, Mueller MD, Taylor RN. Immunobiology of endometriosis. Fertil Steril 2001;75:1-10.

23. Wesselmann U. Neurogenic inflammation and chronic pelvic pain. World J Urol 2001;19:180-185.

24. Wu Z, Yuan M, Li Y, et al. Analysis of WNT4 polymorphism in Chinese Han women with endometriosis. Reprod Biomed Online 2015;30:415-420.

25. Ortiz ME, Croxatto HB. Copper-T intrauterine device and levonorgestrel intrauterine system: biological bases of their mechanism of action. Contraception 2007;75:S16-30.

26. Ortiz ME, Croxatto HB, Bardin CW. Mechanisms of action of intrauterine devices. Obstet Gynecol Surv 1996;51: S42-51. 
27. Stanford JB, Mikolajczyk RT. Mechanisms of action of intrauterine devices: update and estimation of postfertilization effects. Am J Obstet Gynecol 2002;187: 1699-1708.

28. d'Arcangues C. Worldwide use of intrauterine devices for contraception. Contraception 2007;75:S2-7.

29. Diedrich JT, Klein DA, Peipert JF. Long-acting reversible contraception in adolescents: A systematic review and meta-analysis. Am J Obstet Gynecol 2017;216:364 e361-364 e312.

30. Group ECW. Intrauterine devices and intrauterine systems. Hum Reprod Update 2008;14:197-208.

31. Lewis RA, Taylor D, Natavio MF, Melamed A, Felix J, Mishell D Jr. Effects of the levonorgestrel-releasing intrauterine system on cervical mucus quality and sperm penetrability. Contraception 2010;82:491-496.

32. Chaganti RK, Lane NE. Risk factors for incident osteoarthritis of the hip and knee. Curr Rev Musculoskelet Med $2011 ; 4: 99-104$.

33. Jackson KA, Glyn-Jones S, Batt ME, Arden NK, Newton JL, Delphi P. Assessing risk factors for early hip osteoarthritis in activity-related hip pain: A Delphi study. BMJ Open 2015;5:e007609.

34. Shane Anderson A, Loeser RF. Why is osteoarthritis an age-related disease? Best Pract Res Clin Rheumatol 2010;24: 15-26.

35. Okoro CA, Zhao G, Fox JB, Eke PI, Greenlund KJ, Town M. Surveillance for health care access and health services use, adults aged 18-64 years-Behavioral risk factor surveillance system, United States, 2014. MMWR Surveill Summ 2017;66:1-42.
36. Tsui J, Hirsch JA, Bayer FJ, et al. Patterns in geographic access to health care facilities across neighborhoods in the united states based on data from the national establishment time-series between 2000 and 2014. JAMA Netw Open 2020;3:e205105.

37. Bjelland EK, Kristiansson P, Nordeng H, Vangen S, Eberhard-Gran M. Hormonal contraception and pelvic girdle pain during pregnancy: A population study of 91, 721 pregnancies in the Norwegian Mother and Child Cohort. Hum Reprod 2013;28:3134-3140.

38. Sverdrup B, Kristiansson P. Pelvic girdle pain may be an overlooked hormone adverse effect. Acta Obstet Gynecol Scand 2004;83:316-317.

39. Hassan S, Muere A, Einstein G. Ovarian hormones and chronic pain: A comprehensive review. Pain 2014;155: 2448-2460.

40. Kesikburun S, Guzelkucuk U, Fidan U, Demir Y, Ergun A, Tan AK. Musculoskeletal pain and symptoms in pregnancy: A descriptive study. Ther Adv Musculoskelet Dis 2018;10:229-234.

41. Watt FE. Musculoskeletal pain and menopause. Post Reprod Health 2018;24:34-43.

42. Lee YJ, Kim SH, Chung SW, Lee YK, Koo KH. Causes of chronic hip pain undiagnosed or misdiagnosed by primary physicians in young adult patients: A retrospective descriptive study. J Korean Med Sci 2018;33: e339.

43. Roberts WN, Williams RB. Hip pain. Prim Care 1988;15: 783-793.

44. Ward D, Parvizi J. Management of hip pain in young adults. Orthop Clin North Am 2016;47:485-496. 